\title{
A novel strain of cynomolgus macaque cytomegalovirus: implications for host-virus co-evolution
}

\author{
Justen N. Hoffman Russell', Angie K. Marsh', David O. Willer 2,3, Aruna P. N. Ambagala ${ }^{2,3,9}$, Misko Dzamba ${ }^{4}$, \\ Jacqueline K. Chan ${ }^{2,3}$, Richard Pilon ${ }^{5}$, Jocelyn Fournier ${ }^{6}$, Michael Brudno ${ }^{4}$, Joseph M. Antony ${ }^{2,3}$, Paul Sandstrom, \\ Ben J. Evans ${ }^{7}$ and Kelly S. MacDonald ${ }^{1,2,3,8,10^{*}}$
}

\begin{abstract}
Background: Cytomegaloviruses belong to a large, ancient, genus of DNA viruses comprised of a wide array of species-specific strains that occur in diverse array of hosts.

Methods: In this study we sequenced the $\sim 217 \mathrm{~Kb}$ genome of a cytomegalovirus isolated from a Mauritius cynomolgus macaque, CyCMV Mauritius, and compared it to previously sequenced cytomegaloviruses from a cynomolgus macaque of Filipino origin (CyCMV Ottawa) and two from Indian rhesus macaques (RhCMV 180.92 and RhCMV 68-1).

Results: Though more closely related to CyCMV Ottawa, CyCMV Mauritius is less genetically distant from both RhCMV strains than is CyCMV Ottawa. Several individual genes, including homologues of CMV genes RL11B, UL123, UL83b, UL84 and a homologue of mammalian COX-2, show a closer relationship between homologues of CyCMV Mauritius and the RhCMVs than between homologues of CyCMV Mauritius and CyCMV Ottawa. A broader phylogenetic analysis of 12 CMV strains from eight species recovers evolutionary relationships among viral strains that mirror those amongst the host species, further demonstrating co-evolution of host and virus.
\end{abstract}

Conclusions: Phylogenetic analyses of rhesus and cynomolgus macaque CMV genome sequences demonstrate cospeciation of the virus and host.

Keywords: Cynomolgus macaque, Cytomegalovirus, Phylogenetics, Co-speciation, Co-evolution

\section{Background}

Macaque monkeys are an important animal model in biomedical research, particularly in infectious diseases. They are used in vaccine and infectivity studies of viruses that either do not infect or do not produce realistic pathogenic features in small animal models - viruses such as Human Immunodeficiency Virus (HIV) where Simian Immunodeficiency Virus (SIV) or the human envelope substituted version (SHIV) can be studied as a surrogate in macaques.

Recently, the limited availability of Indian rhesus macaques in North America and elsewhere has led to the

\footnotetext{
* Correspondence: kelly.macdonald@umanitoba.ca

'Department of Immunology, University of Toronto, Toronto M5S 1A8ON, Canada

2Department of Medicine, University of Toronto, Toronto M5S 1A8ON, Canada

Full list of author information is available at the end of the article
}

development of resources and standards for the use of cynomolgus macaques and Chinese rhesus macaques [1], the former in particular being an excellent model of HIV-1/SIV infection [2]. Though relatively closely related, divergence between populations or species of macaques and their corresponding cytomegaloviruses (CMV) strains is substantial enough as to preclude direct replacement in any study. In fact, with increased usage in research, it has been questioned whether cynomolgus macaques may be in need of taxonomic reclassification [3]. Captive populations of cynomolgus macaques are frequently interbred with little concern as to their origins. However, the genetic divergence between geographically distinct populations of cynomolgus macaques rivals that found between Indian and Chinese rhesus macaques, a factor that has the potential to differently influence experimental results [3]. For example, 
disease susceptibility is highly variable between isolatedtightly knit groups of macaques found across the Sunda Shelf of Southeast Asia. Macaque genome sequencing has enabled a better understanding of their disease susceptibility from an evolutionary and conservation standpoint, and provided insight into population structure and patterns of migration [3-6].

Interest in nonhuman primate herpesviruses, and CMVs in particular, has increased in recent years in recognition of the unique immune response that they evoke. Specifically, CMV evokes a type of effector memory $\mathrm{T}$ cell response, which intermittently is boosted by reactivated virus throughout the life of its immunocompetent host. This type of immune memory qualitatively is different from conventional $\mathrm{T}$ cell memory in that it does not require priming and can respond immediately to antigen. Thus CMVs are also of interest to vaccinologists who are examining ways to expand the duration of rapidly inducible $\mathrm{T}$ cell responses to block primary viral infection. The evaluation of CMVs in rhesus and more recently, in cynomolgus macaques, has become a priority in order to facilitate the use of these animal models for vaccine research and development. CMV infects many primate and non-primate hosts including humans, baboons, green monkeys, chimpanzees, squirrel monkeys, macaques, oysters and rodents [7]. Since previous studies have demonstrated only limited mixing and horizontal transmission between mammalian populations [8], it is thought that CMV, like other herpesviruses, diversified via co-evolution with their host species $[8,9]$.

During speciation, pathogens may co-speciation with their host [10]. Herpesviruses, for instance, co-evolve with their mammalian hosts [11]. Herpesviruses were present in primates 70 mya [12], and have been used as surrogates to track mammalian - including human evolution and migration [12, 13]. Herpesviruses, including CMVs, were also present in the most recent common ancestor of rhesus and cynomolgus macaques, and are presumed to have undergone geographic differentiation similar to their host species [14]. However, macaques can become infected with multiple strains of CMV and these CMV strains may experience recombination. Hosts co-infected with two CMV strains occasionally release infectious viral particles (shed virus) from multiple strains (dual shedding), but generally have a dominant strain that makes up the majority of shed virus [15]. Interaction among populations of cynomolgus macaques can facilitate the exchange of CMV strains [16] but cross infection between species is generally rare [17].

In the present study, we sequenced a new strain of CyCMV from a cynomolgus macaque from Mauritius and compared it to three other strains - one from a cynomolgus macaque that originated in the Philippines (CyCMV Ottawa) [18], and two from Indian rhesus macaques (RhCMV), RhCMV 68-1 [19] and RhCMV 180.92 [20]. We also characterized variation in gene content among these macaque CMVs. Our results indicate that evolutionary relationships over the complete genomes of cynomolgus CMV strains matches those of their hosts, thereby supporting their co-evolution and also the further use of CMVs in the study of mammalian biogeography and phylogeny. We also found phylogenetic correspondence between CMVs and their hosts in a broader phylogenetic analysis that included several other CMV strains. However, phylogenetic relationships among some genomic regions of the macaque CMVs deviated from the expected relationship, and this can be explained either by intra-strain recombination or alternatively could reflect phylogenetic error.

\section{Results and discussion}

\section{CyCMV Mauritius is a unique CMV from Mauritian cynomolgus macaques}

In the present study, we characterized a novel CMV strain, isolated from a Mauritian cynomolgus macaque, and compared its sequence to that of three other CMV strains, including that of CyCMV Ottawa [18], and two strains from rhesus macaques: RhCMV 68-1 [19] and RhCMV 180.92 [20]. Our analyses indicate that the sequence of CyCMV Mauritius is highly similar to the sequence of CyCMV Ottawa, and also that both are derived from a more recent common ancestor than either is with one of the rhesus macaque strains.

Our assembly of Illumina reads from CyCMV Mauritius produced three contigs that were then connected by Sanger sequencing. The assembly had an average 280X fold coverage that was $217200 \mathrm{bp}$ in length, and had $49.5 \%$ GC content. In comparison, CyCMV Ottawa is 841 bp longer and has a similar GC content (Table 1). RhCMV $68-1$ and RhCMV180.92 are both similar in length and GC content, with RhCMV 180.92 being the shortest and RhCMV 68-1 the longest of the four viruses. Compared to other mammalian CMVs, all macaque CMVs examined are shorter than chimpanzee CMV (CCMV) Heberling strain and shorter on average than Human CMV (HCMV) strains AD169 and HAN1. Of the CMVs that have been sequenced thus far, these four macaque CMVs are most similar in length to Aotine (Owl Monkey) CMV and African Green Monkey CMV.

CyCMV Mauritius and CyCMV Ottawa share $95.3 \%$ identity by linear full genome alignment while RhCMV 68-1 and RhCMV 180.92 share $95.6 \%$ identity. CyCMV Mauritius and RhCMV 180.92 have $87.9 \%$ identity and CyCMV Mauritius and RhCMV 68-1 have 89.7 \% identity. CyCMV Ottawa has 89.8 \% identity with RhCMV 68-1 and $88.2 \%$ identity with RhCMV 180.92 .

Due to the possibility of rearrangements in these viral genomes we evaluated sequence synteny using the 
Table 1 Select sequenced primate CMV strains

\begin{tabular}{llllll}
\hline Virus & Strain & Genbank Asscession & Host Species & Length (bp) & \%GC \\
\hline CyCMV & Mauritius & KP796148 & Cynomolgus Macaque & 217200 & $49.5 \%$ \\
CyCMV & Ottawa & JN227533 & Cynomolgus Macaque & 218041 & 215678 \\
RhCMV & 180.92 & DQ120516 & Rhesus Macaque & $49.5 \%$ \\
RhCMV & $68-1$ & AY186194 & Rhesus Macaque & 221454 \\
OMCMV & S34E & FJ483970 & Owl Monkey & 219474 & $49.0 \%$ \\
GMCMV & Colburn & FJ483969 & African Green Monkey & 219526 & $56.3 \%$ \\
GMCMV & FJ483968 & African Green Monkey & 226205 & $51.2 \%$ \\
CCMV & Heberling & AF480884 & Chimpanzee & 241087 \\
HCMV & AD169 & FJ527563 & Human & 229354 & $50.8 \%$ \\
HCMV & HAN1 & JX512199 & Human & 235006 & $57.2 \%$ \\
\hline
\end{tabular}

progressive Mauve multiple genome alignment algorithm [21]. This analysis reveals a local co-linear region around 160-170 kbp where the RhCMV 68-1 sequence is reversed in comparison to CyCMV Mauritius, CyCMV Ottawa and RhCMV 180.92 (Fig. 1). This region is immediately adjacent to the UL128 to UL130 deletion found in RhCMV 68-1. There are two locally collinear blocks whose order are reversed between rhesus (RhCMV 68-1 and RhCMV 180.92) and cynomolgus (CyCMV Mauritius and CyCMV Ottawa) macaque CMV genomes. Both collinear blocks are short and located immediately upstream of the reversed region in RhCMV 68-1.

\section{Phylogenetic relationships among CMV strains match those of their hosts}

Evolutionary relationships were estimated for CyCMV Mauritius, CyCMV Ottawa, RhCMV 180.92 and RhCMV
68-1 using HCMV AD169 as an outgroup (Fig. 2). Evolutionary relationships among these complete macaque CMV genome sequences match those of their hosts (Fig. 2a). Within the cynomolgus macaque, the viral strains CyCMV Mauritius and CyCMV Ottawa are diverged by 0.036 substitutions per site (sps), whereas within the rhesus macaque, the viral strains RhCMV 180.92 and RhCMV 68-1 are diverged by only 0.017 sps. Thus, divergence between CMV strains of cynomologous macaques is greater than between the CMV strains of rhesus macaques, and this may also be true of the respective host populations.

All four-macaque CMVs share similar genome architecture. Macaque CMV and the CMV of closely related primates, like green monkey CMVs (GMCMVs) Colburn and 2715 , have terminal repeats, as well as unique long and unique short regions in genome structure, but lack the internal repeat regions of other primate CMVs [7].

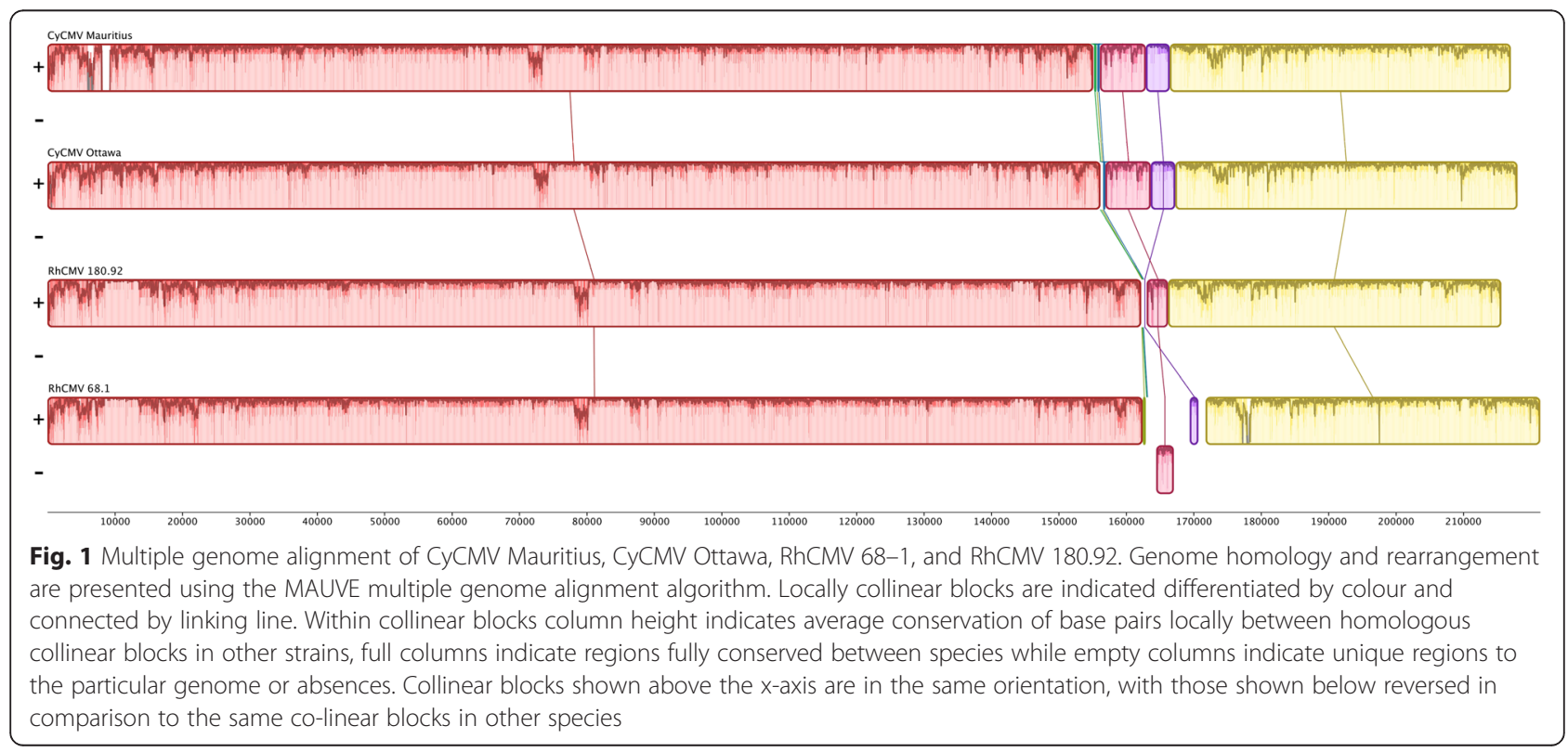




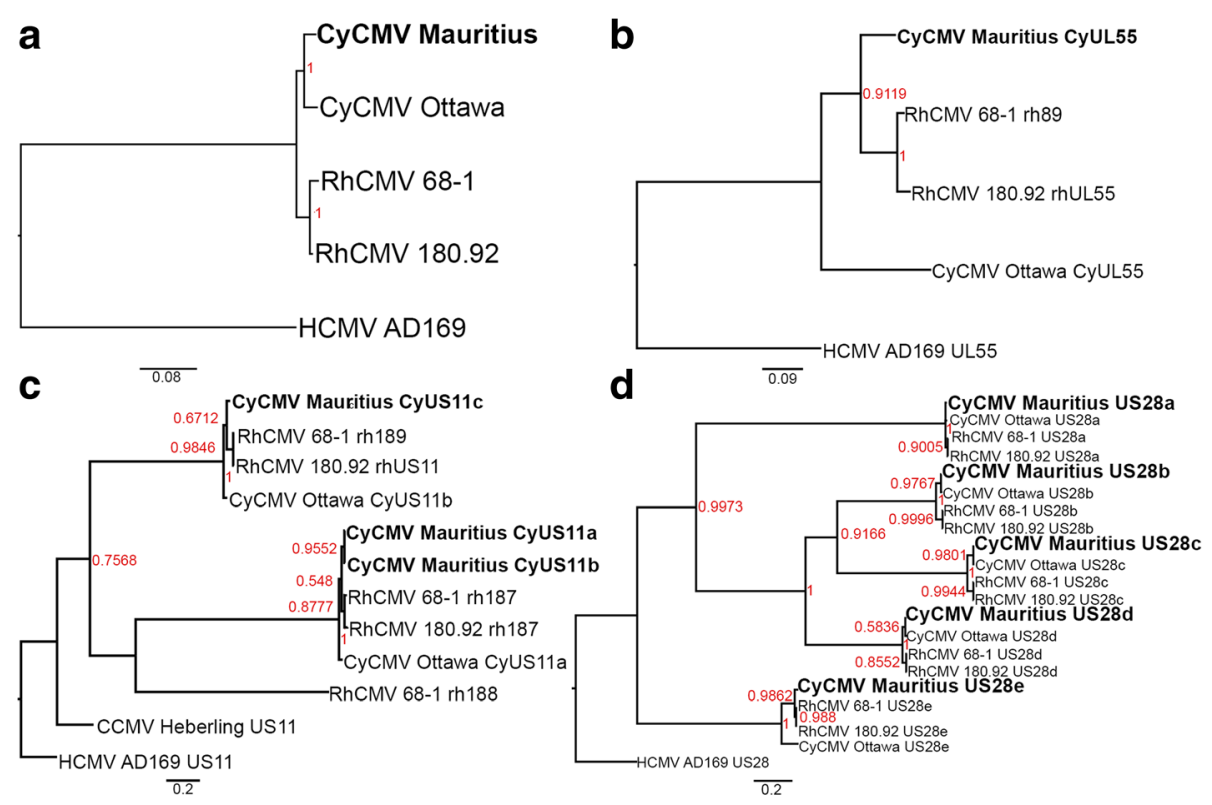

Fig. 2 Phylogenetic tree comparison of Macaque CMV genome and select genes. Trees were generated comparing CyCMV Mauritius, CyCMV Ottawa, RhCMV 68-1 and RhCMV 180.92 using HCMV AD169 as an out-group. Trees were generated using Mr. Bayes, following MAFFT alignment, using a model of evolution selected by JModel test a) from a full genome global alignment; $\mathbf{b}$ ) from an alignment of putative gB (UL55) ORF, a typical marker of phylogeny; c) from an alignment of US11, an MHC-I down-regulatory gene necessary for superinfection of CMV, Chimpanzee CMV is included; $\mathbf{d}$ ) from an alignment of US28 genes, for which 5 copies are encoded in each macaque CMV (4 copies of the gene and one copy of a nonfunctional pseudogene for RhCMV 68-1). CyCMV genes CyUS28a-CyUS28e and RhCMV genes rh214-rh220 are relabeled as US28a US28e, inclusive of the RhCMV 68-1 pseudogene homologous to other macaque CMV US28c, based on order in genome. Numbers at loci indicate posterior probability with color scaled according to probability; genetic distances measured in substitutions per site (sps) are given by scale below

\section{CyCMV genomes show diversity in gene conservation}

Viral genes that are important for host specificity may tend to be more diverged among strains in different hosts. To explore these possibilities, we compared divergence and gene content of the four completely sequenced macaque CMV strains in order to better understand how these factors varied among strains. Annotation of the sequenced CyCMV Mauritius genome identified 290 putative open reading frames (ORFs, Additional file 1: Figure S1; Additional file 2: Table S1). By contrast, CyCMV Ottawa has 262 putative ORFs [18], RhCMV 68-1 has 230 ORFs [19], and RhCMV 180.92 has 258 ORFs [20]. To better understand the conservation of individual ORFs in these CMV genomes we used bit-score, a log-scaled measure indicating the size of a random search string required to find an equivalently or more similar sequence than the observed match. Bit-scores were generated by comparing ORFs in each of the six pairwise comparisons between the four macaque CMV genomes. Bit-scores comparing homologous ORFs of CyCMV Mauritius and CyCMV Ottawa, RhCMV 68-1 or RhCMV 180.92 were examined grouped by gene family (Fig. 3). The average bit-score for genes in each family, but not the bit-score of each individual gene, is higher for the comparison of genes between the two CyCMVs than between CyCMV
Mauritius and RhCMV 180.92 or RhCMV 68-1. The only exception to this is the COX-2 family, which is absent from CyCMV Ottawa.

Two-dimensional bit-score plots were used to further visualize variation in individual gene conservation in the pairwise comparisons between the four macaque CMV strains (Fig. 4), as in [18]. When plotted this way, ORFs found along the $x=y$ diagonal have equivalent sequence conservation in both pairwise comparisons. Genes that are equally well conserved between the two RhCMVs and between the two CyCMVs (Bit Score >1000 in one comparison) cluster along the diagonal. However, this analysis of pairwise comparisons highlights a nonuniform level of sequence conservation in some genes. The cyclooxygenase-2 gene $(C y C O X 2)$, for example, is absent in CyCMV Ottawa. Similarly CyTRL1, an epithelial cell tropism factor [22], is more highly conserved between the RhCMVs than between the two CyCMVs. There was a larger discordance between the bit-scores generated comparing CyCMV genes and those comparing RhCMV for genes with a lower maximum bit-score. Of those genes with known functions, those involved in immune modulation or as temperance factors, and membrane proteins are more dispersed than other groups with many individual genes being more highly conserved in viruses from one species of macaque than 


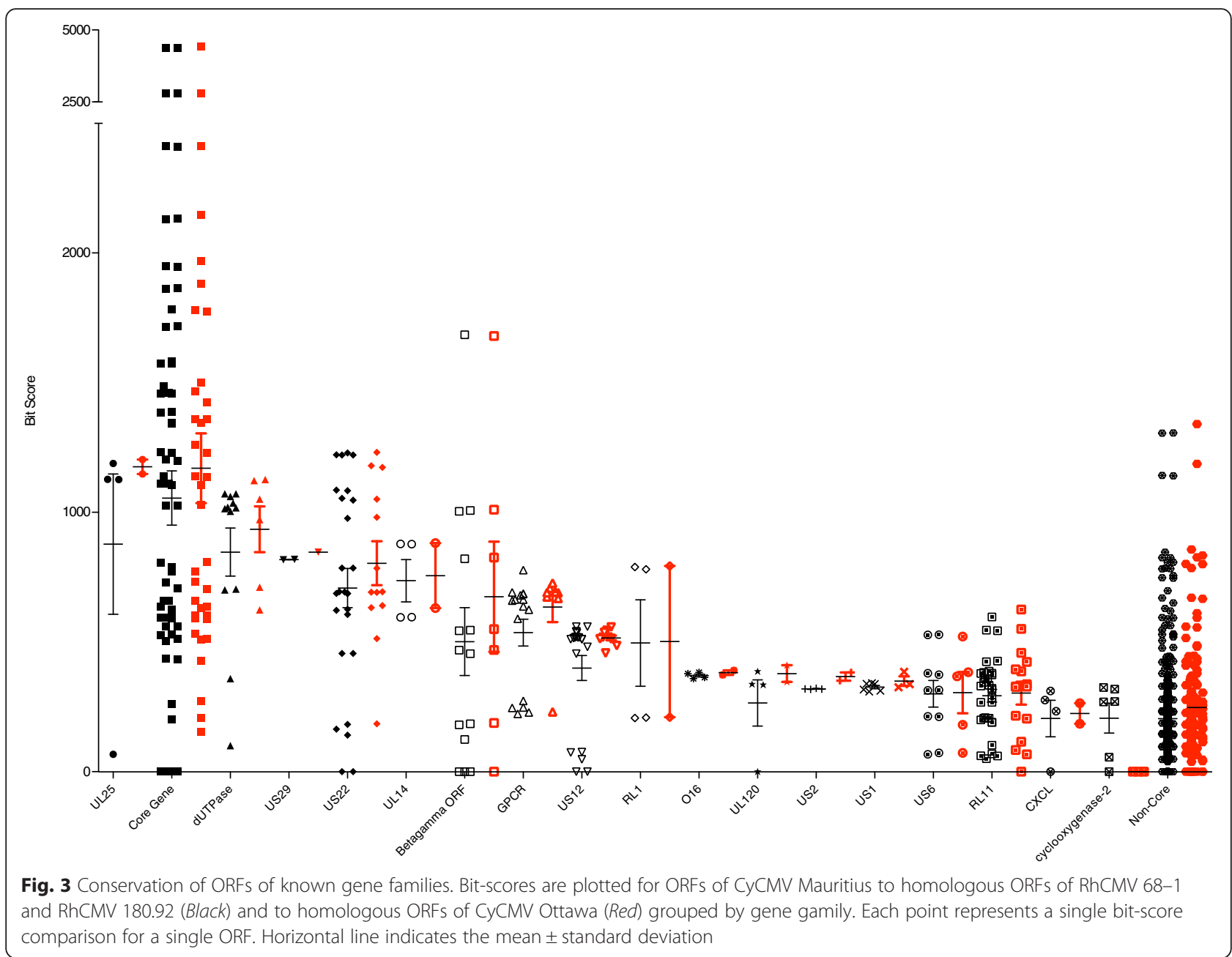

the other. This could suggest that these viral genes face differing evolutionary pressures in the two macaque species.

When grouped by gene function, capsid, DNA binding/nuclear, and tegument groupings contain highly conserved genes and less deviation in bit-score from the diagonal is observed (see Fig. 5; Additional file 3: Figure S2, Additional file 4: Figure S3, Additional file 5: Figure S4, and Additional file 6: Figure S5). In each of these functional categories, genes deviate towards a higher bitscore in CyCMV Mauritius versus CyCMV Ottawa and CyCMV Mauritius versus RhCMV 68-1, and towards a lower bit-score in CyCMV Mauritius versus RhCMV 180.92. A similar trend for higher bit-scores in CyCMV Mauritius versus CyCMV Ottawa and CyCMV Mauritius versus RhCMV 68-1, and a lower bit-score in CyCMV Mauritius versus RhCMV 180.92 was observed with ORFs involved in viral temperance, though the average bit-score was lower, and was observed to a lesser absolute extent with immune regulatory, entry, and membrane associating ORF groupings.
Though closely related to CyCMV Ottawa, CyCMV Mauritius is less genetically diverged from both RhCMVs than CyCMV Ottawa is at several individual genes, including homologues of CMV genes RL11B, UL123, UL83b, UL84 and a homologue of mammalian COX-2 (Additional file 2: Table S1). This suggests a nonuniform rate of evolution of some genes (Fig. 2a) and could reflect differences in selective pressure on the CMV strains in different hosts. The high average bitscore of capsid, DNA binding/nuclear, and tegument ORFs indicate a slow rate of evolution of these genes. Immune regulatory, viral entry, and membrane associated ORFs, in contrast, exhibit signs of more rapid evolution. This may be because the proteins these genes encode interact directly with the host immune system and may consequentially face greater directional or diversifying selection.

Macaque CMV genomes lack an internal repeat region between the unique long (UL) and unique short (US) regions. We explored the effect of this feature of genome architecture on the genomic location of genes using 


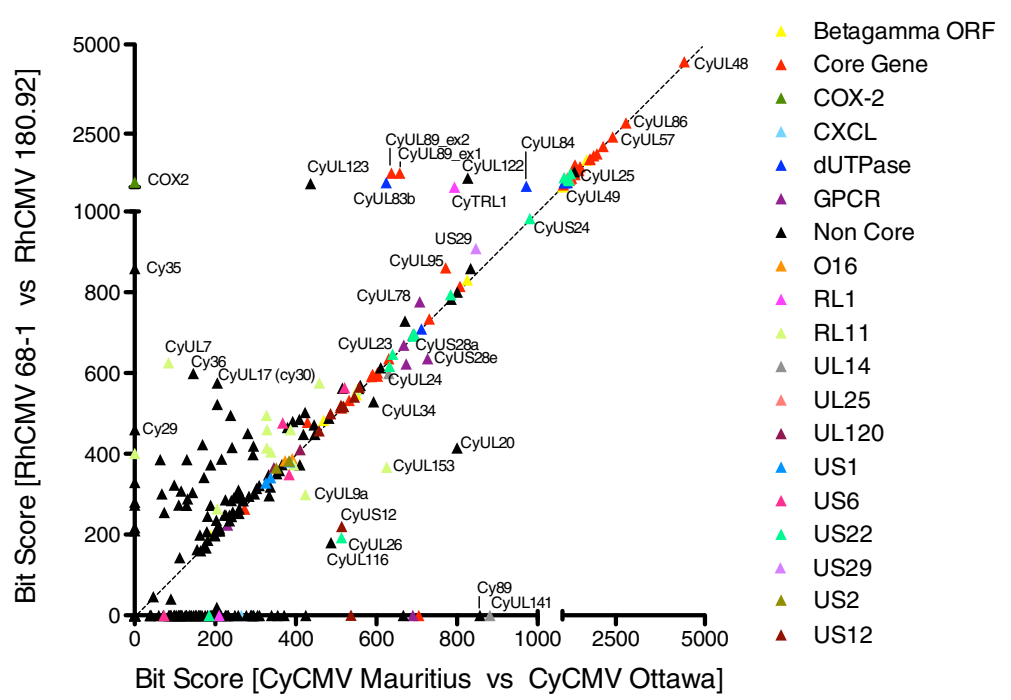

Fig. 4 Two-dimensional bit-score plots between CMV strains. Graph represents comparisons of gene homologue bit-scores across CyCMV Mauritius versus CyCMV Ottawa and RhCMV 68-1 versus RhCMV 180.92. Bit-score among RhCMV homologues of the indicated gene are tracked on the $y$-axis while CyCMV homologues are tracked on the $\mathrm{x}$-axis. A high bit-score indicated a high level of similarity between genes, while a bit-score of zero indicates one or both species in the comparison lacked a copy of the gene. Genes that fall on the diagonal are equally conserved in all comparisons, for example CyUL48 (rh78) and CyUL74 (rh74); genes that fall to one side of the diagonal are better conserved between viruses of one species than between viruses of the other species, for example COX-2 (rh 10) which is highly conserved between RhCMV 180.92 and RhCMV 68-1, but absent from CyCMV Ottawa, and CyUL94 (rh129). Genes are coloured according to known gene family. Select genes are annotated according to their CyCMV Mauritius names. Where genes belong to multiple families only one is indicated, for breakdown of chart by gene family see Additional file 8: Figure S7 or Additional file 9: Figure S8, Additional file 10: Figure S9 and Additional file 11: Figure S10 for alternative pairwise comparisons

gene bit-scores. Our analyses indicate that there is more divergence between genes from different macaque CMV strains that are closer to the terminal regions of both the UL and US genome regions than between genes in different strains that are far from these genomic features
(Additional file 7: Figure S6). This decrease in gene conservation near the UL/US border was observed even in the absence of an internal terminal repeat region between the UL and US regions. Additionally, these terminal areas contain a higher proportion of genes that

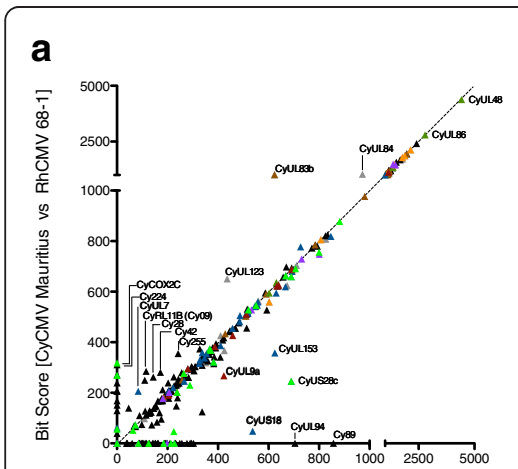

Bit Score [CyCMV Mauritius vs CyCMV Ottawa]

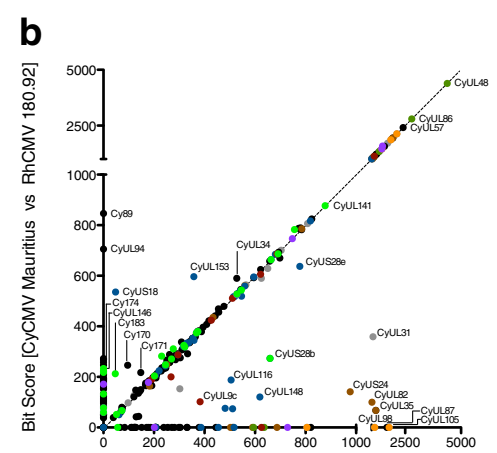

Bit Score [CyCMV Mauritius vs RhCMV 68-1]

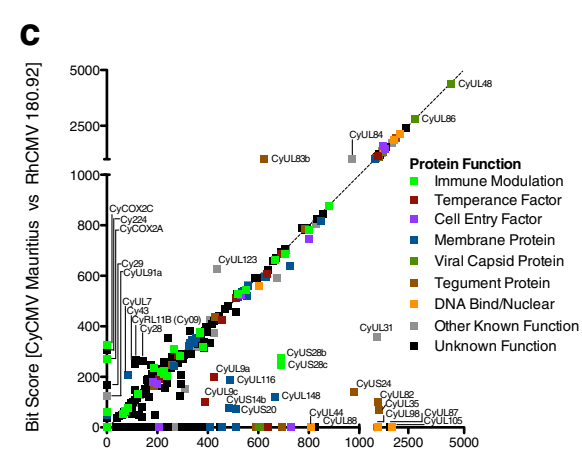

Bit Score [CyCMV Mauritius vs CyCMV Ottawa]

Fig. 5 Two-dimensional bit-score plots between CMV strains. Graphs represent comparisons of gene homologue bit-scores across three strains simultaneously. Comparison of CyCMV Mauritius versus CyCMV Ottawa and CyCMV Mauritius versus RhCMV 68-1 (a), CyCMV Mauritius versus RhCMV 180.92 and CyCMV Mauritius versus RhCMV 68-1 (b), and CyCMV Mauritius versus RhCMV 180.92 and CyCMV Mauritius versus CyCMV Ottawa (c). Genes are coloured according to known function. Immune ORFs are involved in host immune regulation or evasion, entry ORFs are known cell entry factors, temperance ORFs are involved in temperance of viral growth, membrane ORFs are presented in cell membrane on infected cells or in virions, DNA_nuclear are known to interact with DNA or shuttle to the cell nucleus, capsid ORFs form the virus capsid, tegument proteins are found in the viral tegument, the function of ORFs with other known function vary, and the function is unknown at the time of writing for ORFs plotted as unknown. Individual ORF may have several functions; see Additional file 3: Figure S2, Additional file 4: Figure S3, Additional file 5: Figure S4 and Additional file 6: Figure S5 for visualization without overlap. Select genes are annotated according to their CyCMV Mauritius names 
are strain specific. The center of the UL region contains a region with a high density of highly conserved genes. This is expected since in general, the rate of evolution of genes in a virus is slower in key genes, faster in less important genes, and fastest in non-coding and nonregulatory regions $[23,24]$, and because the UL region includes a cluster of core genes that are conserved across all herpesviruses.

During CMV replication, the genome circularizes and large genome segments may be reordered, reversed or lost [25]. This mechanism could explain why the UL128-UL131 region is absent in RhCMV 68-1, but present in RhCMV 180.92 [26], and why rh12-rh16 is absent from CyCMV Mauritius and CyCMV Ottawa but present in both RhCMVs. Loss of the UL128-UL131 region is typical of attenuation (that is, the a decrease in virulence after passaging) in fibroblast cell lines, and demonstrates an inability to infect endothelial and epithelial cell lines. Thus our findings do not necessarily prove an absence of this region in the original wild-type RhCMV 68-1 [7, 19, 27]. How reversal and reordering of CMV genome segments [28] affect the evolution of
CMV in wild macaque populations is yet to be fully understood.

\section{Gene content of CyCMV Mauritius is distinguished from other strains}

Several CMV genes found in CyCMV Ottawa, RhCMV 68-1 and RhCMV 180.92 are absent in CyCMV Mauritius. In particular, nine CyCMV Ottawa ORFs lack homologues in CyCMV Mauritius whereas 20 CyCMV Mauritius ORFs lack homologues in CyCMV Ottawa, and of the latter, nine have homologues that are present in both RhCMV strains (Additional file 2: Table S1). In all, 20 genes of RhCMV 68-1 and RhCMV 180.92 are not identified in CyCMV Mauritius (Table 2). Similarly, comparison to human CMV data, identified 84 ORFs present in strains of HCMV but absent in CyCMV Mauritius (Table 3). The majority of genes absent in all macaque CMV strains but present in HCMV strains have unknown functions though some, such as UL65 and UL108, are known to have effects on CMV growth kinetics (Table 3).

Table 2 RhCMV genes absent from CyCMV Mauritius

\begin{tabular}{|c|c|c|c|c|c|}
\hline Gene & $\begin{array}{l}\text { Alternative } \\
\text { Gene Name }\end{array}$ & $\begin{array}{l}\text { Human } \\
\text { Homologue }\end{array}$ & $\begin{array}{l}\text { Necessary for } \\
\text { growth in Towne }\end{array}$ & $\begin{array}{l}\text { Rh-Human } \\
\text { Similarity }(\%)^{b}\end{array}$ & Function $^{c}$ \\
\hline Rh9 & - & - & - & - & \\
\hline Rh12 & RL11F & - & - & - & \\
\hline Rh13 & RL11G & - & - & - & \\
\hline Rh13.1 & - & - & - & - & \\
\hline Rh14 & - & - & - & - & Membrane protein \\
\hline Rh15 & - & - & - & - & \\
\hline Rh16 & - & - & - & - & \\
\hline Rh17 & $\mathrm{RL} 11 \mathrm{H}$ & UL11 & - & - & early glycoprotein \\
\hline Rh18 & - & - & - & - & \\
\hline Rh19 & RL11। & UL07 & - & $34 \%$ & Membrane protein \\
\hline Rh38 & - & - & - & - & \\
\hline Rh45 & - & - & - & - & \\
\hline Rh77 & - & - & - & - & \\
\hline Rh94 & - & - & - & - & \\
\hline Rh96 & - & - & - & - & \\
\hline Rh121 & - & - & & - & \\
\hline Rh129 & RhUL94 & UL94 & essential & $64 \%$ & Virion Protein \\
\hline Rh142.2 & - & - & - & - & \\
\hline Rh151.1 & - & - & - & - & \\
\hline Rh158 & RhUL147 & UL147 & dispensable & - & Viral CXC Chemokine homologue \\
\hline
\end{tabular}

Footnotes:

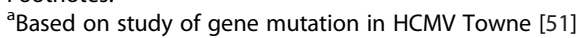

${ }^{b}$ Similarity annotation based on comparisons of RhCMV 68.1 and HCMV in [19]

${ }^{\mathrm{c}}$ Function annotated based on studies of HCMV [52] 
Table 3 Human genes absent from CyCMV Mauritius

\begin{tabular}{|c|c|c|c|c|}
\hline Gene & $\begin{array}{l}\text { CyCMV Ottawa/RhCMV } \\
68.1 \text { Homologue }\end{array}$ & Function $^{a}$ & $\begin{array}{l}\text { Effect of deletion on viral } \\
\text { growth kinetics }\end{array}$ & Family \\
\hline $\mathrm{RL2}$ & $-/-$ & - & no effect & \\
\hline RL3 & $-/-$ & - & no data & \\
\hline RL4 & $-/-$ & - & no effect & \\
\hline RL5 & $-/-$ & - & no data & RL11 \\
\hline RL6 & $-/-$ & - & no effect & RL11 \\
\hline RL7 & $-/-$ & - & no data & \\
\hline RL8 & $-/-$ & - & no data & \\
\hline RL9 & $-1-$ & - & no effect & \\
\hline RL10 & $-/-$ & - & no effect & RL11 \\
\hline RL11 & cyRL11/rh05 & IgG Fc-binding glycoprotein & no effect & RL11 \\
\hline RL12 & $-/-$ & Putative membrane glycoprotein & no effect & RL11 \\
\hline RL13 & $-/-$ & Putative membrane glycoprotein & no effect & \\
\hline RL14 & $-/-$ & - & no data & \\
\hline UL1 & $-/-$ & - & no data & RL11 \\
\hline UL2 & $-/-$ & - & modest effect & RL11 \\
\hline UL3 & $-/-$ & - & no effect & \\
\hline UL4 & $-/-$ & - & no effect & RL11 \\
\hline UL5 & $-/-$ & - & no effect & RL11 \\
\hline UL8 & $-/-$ & - & no effect & RL11 \\
\hline UL10 & $-/-$ & Temperance factor in retinal tissue ${ }^{b}$ & no effect & RL11 \\
\hline UL12 & $-/-$ & - & modest effect & \\
\hline UL15 & $-1-$ & - & no effect & \\
\hline UL16 & $-1-$ & Membrane glycoportein involved in inhibiting Natural Killer cell cytotoxicity & no effect & \\
\hline UL17 & $-/$ rh35 & 7-transmembrane glycoprotein & no effect & \\
\hline UL18 & $-/-$ & $\mathrm{MHCl}$ homologue putative membrane protein & no effect & UL18 \\
\hline UL22 & $-1-$ & - & no data & \\
\hline UL39 & $-1-$ & - & no effect & \\
\hline UL40 & $-/$ rh67 & Membrane glycoptrotein & no data & \\
\hline UL58 & $-/-$ & - & no data & \\
\hline UL59 & $-/-$ & - & no effect & \\
\hline UL60 & $-/-$ & - & required for replication & \\
\hline UL61 & $-1-$ & - & no data & \\
\hline UL62 & $-/-$ & - & no effect & \\
\hline UL63 & $-/-$ & - & no data & \\
\hline UL64 & $-1-$ & - & no effect & \\
\hline UL65 & $-/-$ & - & modest effect & \\
\hline UL66 & $-1-$ & - & no data & \\
\hline UL67 & $-/-$ & - & no effect & \\
\hline UL68 & $-/-$ & - & no data & \\
\hline UL80.5 & -/rh109.1 & Capsid Scaffold Protein & no data & Core \\
\hline UL81 & $-/-$ & - & no data & \\
\hline UL101 & $-/-$ & - & required for replication & \\
\hline
\end{tabular}


Table 3 Human genes absent from CyCMV Mauritius (Continued)

\begin{tabular}{|c|c|c|c|c|}
\hline UL106 & $-/-$ & - & no data & \\
\hline UL107 & $-/-$ & - & no data & \\
\hline UL108 & $-/-$ & - & no data & \\
\hline UL109 & $-/-$ & - & modest effect & \\
\hline UL110 & $-/-$ & - & no effect & \\
\hline UL118 & $-/$ rh151 & - & no effect & \\
\hline UL124 & -/rh156.2 & Membrane glycoprotein latent protein & no data & \\
\hline UL125 & $-/-$ & - & variable critical effect & \\
\hline UL127 & $-/-$ & - & no data & \\
\hline UL129 & $-/-$ & - & no effect & \\
\hline UL143 & $-/-$ & Inhibits Natural Killer cell cytotoxicity & modest effect & \\
\hline UL142 & $-/-$ & Putative membrane protein & no data & \\
\hline UL139 & $-/-$ & Putative membrane glycoprotein & no data & \\
\hline UL138 & $-1-$ & Putative membrane protein & no data & \\
\hline UL137 & $-1-$ & - & no data & \\
\hline UL136 & $-/-$ & Putative membrane protein & no data & \\
\hline UL135 & $-/-$ & Putative secreted protein & no data & \\
\hline UL134 & $-/-$ & - & no data & \\
\hline UL133 & $-1-$ & Putative membrane protein & no data & \\
\hline UL148A & $-1-$ & Putative membrane protein & no data & \\
\hline UL148B & $-/-$ & Putative membrane protein & no data & \\
\hline UL148C & $-/-$ & Putative membrane protein & no data & \\
\hline UL148D & $-/-$ & Putative membrane protein & no data & \\
\hline UL149 & $-/-$ & & no data & \\
\hline UL150 & $-/-$ & Putative Secreted Protein & no data & \\
\hline IRS1 & $-1-$ & $\begin{array}{l}\text { Immediate early membrane protein \& transcriptional activator/blocks protein } \\
\text { kinase R mediated repression of translation }\end{array}$ & no data & \\
\hline US4 & $-/-$ & - & no effect & US22 \\
\hline US5 & $-/$ rh183 & - & no data & \\
\hline US6 & $-/$ rh185 & Putative membrane glycoprotein/Inhibits TAP mediated ER peptide transport & no data & \\
\hline US7 & $-1-$ & Membrane glycoprotein & no effect & US6 \\
\hline US8 & /-rh187 & MHCl binding Membrane glycoprotein & no effect & US6 \\
\hline US9 & $-/-$ & Membrane glycoprotein involved in cell to cell spread & no effect & US6 \\
\hline US10 & $-/-$ & membrane glycoprotein/Delays trafficking of $\mathrm{MHCl}$ & no effect & US6 \\
\hline US15 & $-1-$ & Putative multiple transmembrane protein & no effect & US6 \\
\hline US16 & $-/-$ & Temperance factor and Putative multiple transmembrane protein & no effect & US12 \\
\hline US25 & $-/-$ & - & no effect & US12 \\
\hline US27 & $-/-$ & Virion Envelope Glycoprotein/Potentiates CXCR4 receptor ${ }^{c}$ & no effect & \\
\hline US33 & $-/-$ & - & no effect & GPCR \\
\hline US34 & $-1-$ & Putative secreted protein & no effect & \\
\hline US34A & $-/-$ & Putative membrane protein & no effect & \\
\hline
\end{tabular}

Footnotes:

${ }^{\text {a }}$ Function annotated based on studies of HCMV [52] unless otherwise indicated

${ }^{b}$ Based on study of gene mutation in HCMV Towne [51]

'Based on study of US27 in HCMV AD169 [53] 


\section{Anomalous gene trees reveal patterns of gene family evolution}

Analysis of individual genes reveals several interesting features of these viral genomes. The Bayesian phylogeny for the important viral surface glycoprotein B gene (UL55), for example, indicates an atypically high level of divergence among these strains as compared to the whole genomes (Fig. 2b). This is of interest since it is a target of immune responses. In the analysis of the UL55 gene, CyCMV Mauritius and CyCMV Ottawa do not cluster together, and CyCMV Mauritius instead is inferred to be more closely related to the RhCMVs than to CyCMV Ottawa. CyCMV Mauritius UL55 is diverged from CyCMV Ottawa by 0.408 sps but diverged from RhCMV 68-1 and RhCMV 180.92 by only 0.175 and 0.188 sps respectively. Figure $2 c$ shows a phylogenic estimate for the US11 gene, which encodes an MHC down regulatory protein essential for superinfection [29]. Included in the analysis are multiple gene homologs of US11 generated by gene duplication in various CMVs. Inferred evolutionary relationships within US11 paralogs again indicate a closer evolutionary relationship between CyCMV Mauritius and the RhCMVs than to CyCMV Ottawa, although with weak statistical support (87.7\% posterior probability). Interestingly, rh188 is not present in RhCMV 180.92.

Figure $2 \mathrm{~d}$ shows results of a phylogenetic analysis of the multiple copies of US28, a macaque CMV capsid protein that induces COX-2 in target cells upon entry. CyCMV Mauritius genes were named following the synteny-based system established for CyCMV Ottawa [18]. The five copies of CyCMV Ottawa, CyCMV Mauritius and RhCMV 180.92, as well as the four copies and single pseudogene of RhCMV 68-1, cluster first by synteny and then by species. This likely indicates that divergence of all five copies occurred before speciation and that loss of one copy in RhCMV 68-1 occurred after speciation. Unlike the other US28 genes, however, phylogenetic sequence analysis of US28e (rhUS28) in RhCMV180.92, rh220 in RhCMV 68-1, CyUS28e (in both CyCMVs) suggests that CyCMV Mauritius and CyCMV Ottawa are each more closely related to RhCMV US28e than to CyCMV US28e. CyCMV Mauritius is diverged from CyCMV Ottawa by 0.175 sps and diverged from RhCMV 180.92 and RhCMV 68-1 by 0.031 sps and 0.031 sps, respectively. CyCMV Ottawa is diverged from RhCMV 180.92 and RhCMV 68-1 by 0.168 sps and 0.169 sps, respectively.

Phylogenetic relationships were further estimated for several CMV genes known to have homology to mammalian host genes (Fig. 6). Strain specific absence of several human and rhesus homologues supports independent evolution of each strain. This is evidenced, for example, by the absence of the COX-2 gene in CyCMV
Ottawa. Similarly, a COX-2 homologue (rh10, CyCOX2, this study), which promotes the formation of arachidonic acid in infected cells [30], is present in CyCMV Mauritius, RhCMV 180.92 and RhCMV 68-1, but absent in CyCMV Ottawa [30]. Viral COX-2 (rh10) is necessary for the infection of endothelial cells [30] and it has been shown that viral COX-2, but not cellular COX2 , protein is expressed when RhCMV 68-1 infects cells [30]. Since CMV has been implicated in vascular inflammation [31-33], the study of rh10, which confers cellular tropism and its selection in macaques, is of particular interest. US 28 is a viral capsid protein capable of inducing host COX-2 expression in target cells [34]. CyCMV Mauritius, CyCMV Ottawa and RhCMV 180.92 have five copies each while RhCMV 68-1 has only four copies of US28 genes and a US28 pseudogene. The pseudogene in RhCMV 68-1 shows that it, similar to other macaque CMVs, had five copies of US28, and that the fifth copy was likely pseudogenized after speciation. The number of copies of US28 is variable across mammalian CMVs. Baboon CMV, similar to macaque CMV, has four distinct copies, HCMV has a single copy and both RCMV and MCMV lack US28 homologues [35, 36]. These multiple copies are more divergent from each other within a genome than they are from corresponding homologues between macaque species (Fig. 2d) and can be assumed to have emerged prior to the speciation of these macaques.

COX-2 homologues are absent from CyCMV Ottawa and HCMV but present in both RhCMV 68-1 and RhCMV 180.92 [18, 19, 30, 34] Interestingly, however, the CyCMV Mauritius genome has three ORFs with homology to COX-2: $C y C O X-2 A, C y C O X-2 B$, and $C y C O X-2 C$. It is perhaps not coincidental that RhCMV 68-1, which has a COX-2 homologue the least diverged from mammalian COX-2, is also the strain with fewest functioning US28 genes. Meanwhile, a high degree of divergence between COX-2 homologues in the three strains that retain it suggests a more recent integration or only a low fitness advantage. The absence of the COX-2 homologue in CyCMV Ottawa and the fact that RhCMV 68-1 and CyCMV Mauritius are genetically less distant than RhCMV 68-1 and CyCMV Ottawa may again suggest a faster rate of divergence of CyCMV Ottawa. Comparison of COX-2 homologues (vCOX-2) showed a clustering of CMV homologues that are substantially diverged from their macaque homologs, prostaglandin endoperoxide synthase 2 (PTGS2) in Macaca fascicularis and Macaca mulatta (Fig. 6a). There is no HCMV equivalent of PTGS2. It can be inferred that the $C O X-2$ gene was copied from a mammalian host once to an ancestral CMV prior to CyCMV and RhCMV speciation. A mammalian origin of the vCOX-2 genes is further evidenced by the presence of introns in these genes. 


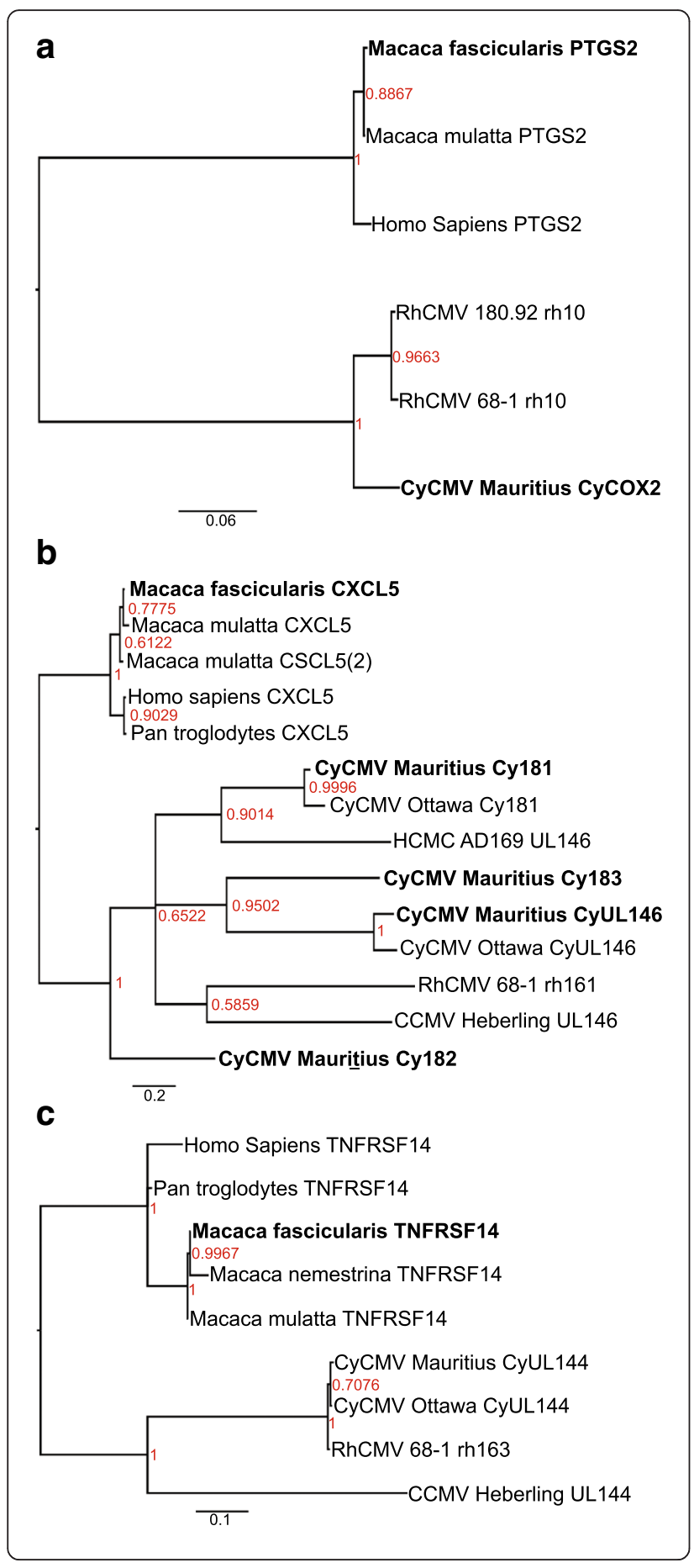

Fig. 6 Phylogenetic tree comparison of CMV genes with mammalian homologues. Trees were prepared comparing CyCMV Mauritius, CyCMV Ottawa, RhCMV 68-1, RhCMV 180.92, CCMV Heberling and HCMV AD169 with mammalian homologue genes using the relevant Homo sapiens homologue as the outgroup. Trees were generated by Mr. Bayes following MAFFT alignment using a model of evolution selected by JModel test comparing a) CyCOX-2/rh10 putative protein product to its mammalian homologue Prostaglandin-endoperoxide synthase 2 (PTGS2); b) UL146 and UL146-like CMV genes with mammalian homologue CXC chemokine ligand 5 (CXCL5); c) UL144 with mammalian homologue tumor necrosis factor receptor superfamily 14 (TNFRSF14). Numbers at loci indicate posterior probability with color scaled according to probability; genetic distances measured in substitutions per site (sps) are given by scale below

Perhaps the unique MHC haplotypes of Mauritian macaques favor the retention of an immune-modulatory gene, such as COX-2 homologue in CyCMV Mauritius.

Similarly, mammalian CXC chemokine ligand 5 (CXCL5) genes substantially diverged from CMV encoded UL146like genes (Fig. 6b). Interestingly, viral chemokine-encoding UL146-like genes such as CCMV UL146 clustered with RhCMV rh161, whereas HCMV UL146 clustered with CyCMV Cy181 with weak support. Two interesting observations were noted. First, both CCMV UL146 and HCMV UL146 are more diverged from mammalian CXCL5 genes (closest is 2.075 sps and 2.069 sps respectively) than they are from multiple macaque CMV genes (closest are 1.299 sps and 1.870 sps respectively). Second, the CyCMV Mauritius gene Cy182 is less diverged from all the mammalian CXCL5 genes than it is from any CMV genes (the closest mammalian gene is Macaca mulatta CXCL5 at 1.234 sps, and the closest macaque gene is CyCMV Mauritius Cy181 1.448 sps). This probably reflects variation in the rate of evolution among these genes and among CMV strains, with the CyCMV Mauritius being slower than the others (Fig. 6b). The clustering of CCMV and HCMV with other CMVs may indicate the acquisition of a viral CXCL5 homologue in a common ancestral CMV strain followed by gene duplication and mutation to create the UL146-like genes. This phylogeny supports at least two independent gene duplication events before diversification of Old World Primates. Possibly after this, other gene duplication events generated additional gene copies in macaques. Alternatively, more than two gene duplication events occurred in the ancestor of Old World primates followed by more extensive gene loss in apes than macaques. It is interesting that no UL146-like genes were identified in RhCMV180.92. Figure $6 \mathrm{c}$ shows a well supported close relationship between CMV UL144 genes of CyCMV Mauritius, CyCMV Ottawa, RhCMV 68-1 and CCMV Heberling to the exclusion of the homologous tumor necrosis factor receptor superfamily 14 (TNFRS14) genes in their macaque and human hosts, which is again consistent with a single copying event in the ancestor of these CMV strains. 
CMVs and their hosts share similar evolutionary patterns In order to test for co-evolution between host species and the CMVs that they harbor over a broader phylogeny, we performed a phylogenetic analysis of 12 available primate CMV viral genomes (Fig. 7). The resulting phylogenetic tree of these CMVs was then compared to a previously estimated phylogenetic tree of mammalian genomes [37]. Phylogenetic relationships among multiple primate CMVs exhibit an identical pattern of CMV and host diversification (Fig. 7); [37]. There is evidence of attenuation in some sequenced CMVs caused by growth of the isolated strains in tissue culture prior to sequencing. This can result in the mutation or loss of viral genes necessary for entry or growth in certain cell types, as is the case with the deletion of the UL128UL130 region in RhCMV 68-1 [38], and other laboratory adapted CMV strains $[39,40]$. Despite this, there is no indication of cross species contact or attenuation of some strains during CMV diversification prior to laboratory isolation. DNA polymerase sequence comparisons have suggested a speciation for CyCMV Ottawa and RhCMV 68-1 of around 0.5 mya [7]. This places speciation and divergence from a common CMV strain at $\sim 1.2$ mya, in line with Y-DNA segregation and the suspected end to macaque migration between islands of the Sunda shelf $[41,42]$.

It is possible for multiple CMV strains to co-exist within a single macaque, or within a single macaque population, because CMV can superinfect seropositive individuals [43]. However, co-infection of a host may only be possible for closely related CMV strains - cynomolgus macaques, for example, are not susceptible to infection or co-infection by a RhCMV [17]. The correspondence between viral and host evolutionary relationships suggests further study of CMV would serve as a molecular tool to understand primate evolution.

\section{Conclusions}

In this study, we generated a novel genome sequence from a CMV strain isolated from a cynomolgus macaque from Mauritius. When analyzed with other CMV genomes from macaques and from other host species, inferred phylogenetic relationships among the viruses generally matched those among their hosts. Comparisons of this new genome with other macaque CMVs identify several functional categories of genes with atypically high levels of divergence, variation in gene content, and several genes with inferred phylogenetic relationships that differed from the genome-wide estimate. These results have implications for use of CMV as a vaccine vector and molecular tool, in CMV pathogenesis studies, as well as providing a tool to assist in tracing both viral and macaque migration and distribution.

\section{Methods}

\section{Isolating virus}

Ethics approval was obtained for all animal procedures through the Health Canada National Nonhuman Primate Animal Care Committee. Urine samples were collected from a 15 year-old cynomolgus macaque imported from Mauritius using bladder catheterization. After filtration through a $0.45 \mu \mathrm{m}$ filter, the urine samples were

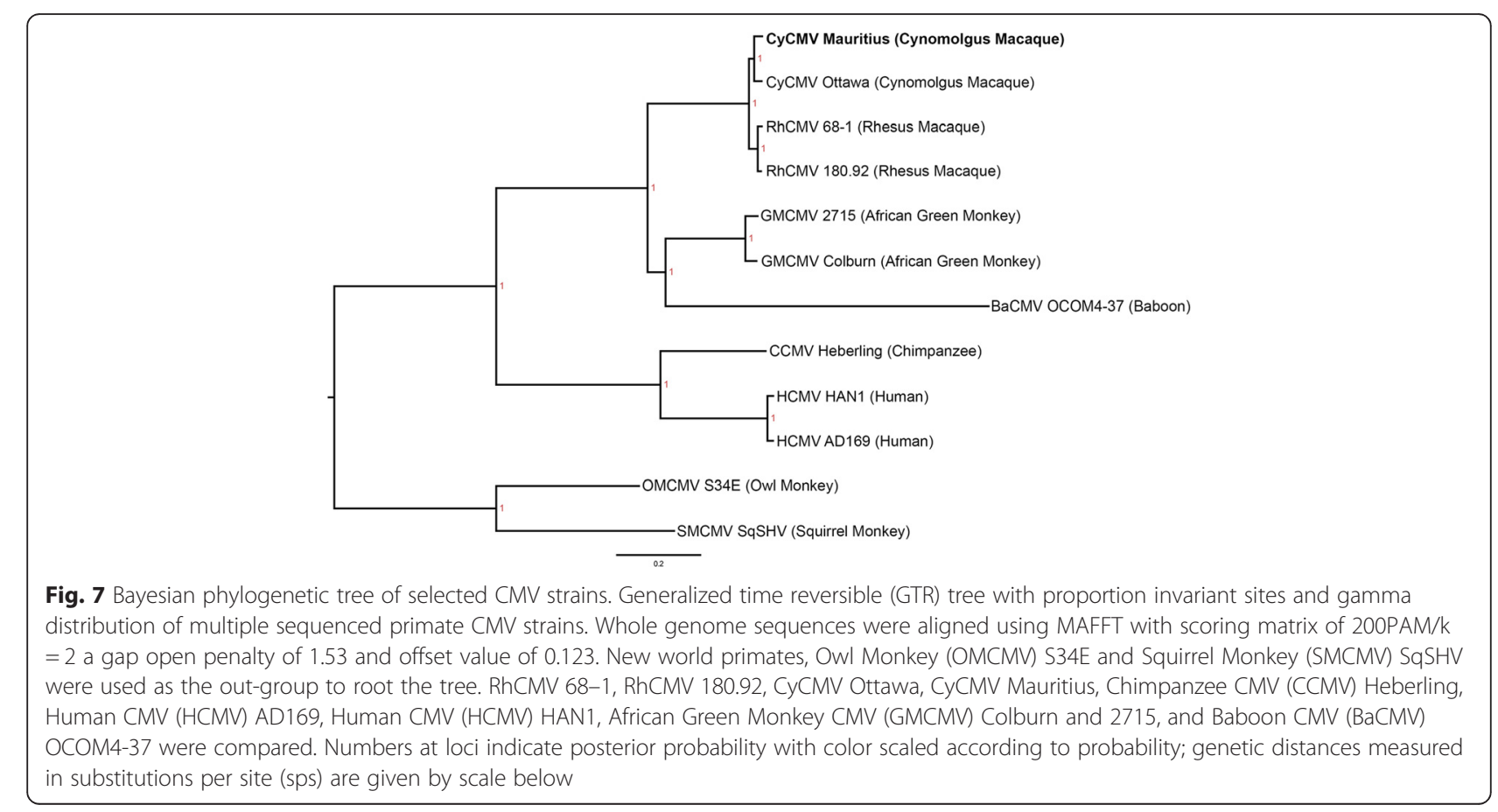


centrifuged at $900 \times \mathrm{g}$ for $30 \mathrm{mins}$ at $4{ }^{\circ} \mathrm{C}$ and supernatants were collected and mixed 1:1 with 2X MEM (supplemented with $2 \mathrm{X}$ antibiotic-antimycotic and $20 \mathrm{mg} / \mathrm{ml}$ gentamycin; Invitrogen). The cell pellets were resuspended with $1 \mathrm{ml}$ of Dulbecco's Modified Eagle's Medium (DMEM; Sigma) supplemented with $1 \mathrm{X}$ antibioticantimycotic (Gibco), and $10 \mathrm{mg} / \mathrm{ml}$ gentamycin (Invitrogen). Urine supernatants were ultracentrifuged at $20000 \mathrm{x}$ $\mathrm{g}$ for $30 \mathrm{mins}$ at $4{ }^{\circ} \mathrm{C}$ and the pellets resuspended in $500 \mu \mathrm{l}$ of DMEM (supplemented with $1 \mathrm{X}$ antibioticantimycotic and $10 \mathrm{mg} / \mathrm{ml}$ gentamycin).

CyCMV Mauritius virus was grown on human fetal lung fibroblast (MRC-5) cells [44] from the American Type Culture Collection (ATCC) as described previously [18]. MRC-5 cells were seeded 1:2 in a 12-well tissue culture plate and grown at $37{ }^{\circ} \mathrm{C}$ in a $5 \% \mathrm{CO}_{2}$ incubator for 2 days (until the cell confluence reached near 100 $\%)$ prior to inoculation. Resuspended viral pellets were each plated in triplicate in wells of MRC- 5 cells, spinoculated at $2000 \times \mathrm{g}$ for $30 \mathrm{mins}$ at $4{ }^{\circ} \mathrm{C}$, and incubated for $2-3 \mathrm{~h}$ at $37{ }^{\circ} \mathrm{C}$ in a $5 \% \mathrm{CO}_{2}$ incubator. The inoculum was aspirated and replaced with $2 \mathrm{ml}$ of DMEM [supplemented with $10 \%$ FBS (Wisent Bioproducts), 100 $\mathrm{U} / \mathrm{ml}$ penicillin, $100 \mu \mathrm{g} / \mathrm{ml}$ streptomycin (Sigma)] and amphotericin $\mathrm{B}$. The medium was changed the following day and every week thereafter. Cultures were monitored daily for CMV cytopathic effect (CPE). Cells showed $100 \%$ CPE after approximately 1 month. The virus was further propagated in MRC-5 cells at a 1:2 split ratio each time CPE reached $90-100 \%$ to achieve higher viral titers.

\section{DNA extraction}

Viral DNA was isolated from cell free virus following centrifugation, digestion with pronase, and phenol-chloroform extraction. Fifteen $75-\mathrm{cm}^{2}$ tissue culture flasks containing CyCMV Mauritius-infected MRC-5 cells were used for viral DNA isolation using a Hirt extraction protocol as described previously [18]. To confirm the purity of the viral DNA isolation, $1 \mu \mathrm{g}$ of isolated extracellular viral DNA was digested with $20 \mathrm{U}$ of BamHI or HindIII restriction enzymes (New England Biolabs) and fractionated by gel electrophoresis on a $0.8 \%$ agarose gel.

\section{Next Generation Sequencing (NGS)}

A paired-end library of CyCMV Mauritius DNA was prepared with $500 \mathrm{bp}$ insert size and high-throughput Illumina Genome Analyzer II paired end sequencing with 72 bp read length was performed as described previously [18]. The complete genome was sequenced at the Centre of Applied Genomics, Toronto, Canada.

\section{Genome assembly}

De novo assembly of the CyCMV Mauritius genome was achieved from 2268822 reads on the Illumina Genome
Analyzer II platform. Paired ends were filtered to match the barcode (1 134411 paired reads) and assembled using Velvet (version 0.7.55) [45]. Results were best obtained using a kmer length of 39 , an insert length of 500 , and an expected coverage of $280 \mathrm{X}$ to generate multiple partial contigs. Three seed sequences, A: 5'-AAACCAGCGCCGTTGTTTTCCGTTCTACGTTCGGG-3', B: 5'-ACTATCGAGGACAACGATGTTTTTTCCAACATA AA-3' and C: 5'-TATCGGTATCTATTCCAAGCAGACC AAGTACGATT-3' were used to create contigs.

\section{Gap closing}

Gap closing in the de novo genome build was accomplished using PCR. Primers were designed from the sequenced contigs, and amplified using purified viral DNA. The resulting product sizes were used to confirm the de novo DNA assembly.

\section{Error correction}

Regions of low resolution or ambiguity were confirmed using Sanger sequencing. First, a 3555 bp region (3 567-7 $122 \mathrm{bp)}$ was confirmed using the primers 5 '-TC GGCAAAGTCAGGAGCGGC-3' and 5'-TGCACAATT TGCGATGCCTATCGTT-3'. Next, Sanger sequencing to amplify the $1207 \mathrm{bp}$ region spanning the origin of lytic replication (OriLyt) [82 561 bp-83 768 bp] was attempted using the primers 5'-TGGCGATCTGAAACC ACACCCC-3' and 5'-CGCCCAAGAGAGAGCGCACC-3' but proved challenging due to the presence of inverted and repeated sequence motifs [46] as our previous experience has shown [18]. Amplification of a 1688 bp segment spanning $154169 \mathrm{bp}-155856 \mathrm{bp}$ using multiple primers also proved unsuccessful. However, a 560 bp region spanning 173964 bp - 174 520 bp was amplified using the primers 5'-ACTTCGCTTCTGTTCTAGCGTTTAGG-3' and 5'-CCGCTGTGGCTTGCTGGCTC-3' and successfully confirmed by sequencing. The CyCMV Mauritius sequence was finally confirmed for errors by aligning with that of RhCMV 68-1 (accession: AY186194), RhCMV 180.92 (accession: DQ120516) and CyCMV Ottawa (accession: JN227533).

\section{Open Reading Frame (ORF) assignment}

1408 putative ORFs, greater than 30 amino acids in length, and not contained within and in frame of another identified potential ORFs were identified in silico using Geneious Pro 6.1.4 (Biomatters Ltd., Auckland, New Zealand). BLAST-P (NCBI) with a BLOSUM62 matrix, gap opening cost of 11 and gap extension cost of one was used to screen the ORFs. Homologous protein lists were generated using a maximum $\mathrm{E}$ value of $10^{-1}$. ORFs with irrelevant or no homology were not included. 


\section{Nomenclature}

Putative genes were named with respect to homologues, as determined above, when applicable. Homologues of human genes are given the prefix ' $\mathrm{Cy}$ ' followed by the names, terminal region long (TRL), terminal region short (TRS), unique long (UL), or unique short (US) and a number previously attributed to the homologue. Homologues of old world monkey CMV genes were given the prefix 'Cy' followed by 'O' and a gene number attributed to the homologue. Some of these were given alternative names, indicated in brackets, due to naming of CyCMV Ottawa homologues prior to this nomenclature [18]. Putative genes with previously named CyCMV Ottawa homologues were given the prefix ' $\mathrm{Cy}$ ' followed by the previously assigned number. Putative genes lacking a CMV homologue were given the prefix ' $\mathrm{Cy}$ ' followed by a unique number not used in naming within CyCMV Ottawa and corresponding to gene order within the genome. Capital letter suffixes (for example, CyUL48A) indicate unique ORFs with a shared gene number as previously established [18]. Lower case single letter suffixes (for example CyUS28b) indicate closely related or repeated genes that share homologous partners. Putative genes were assigned to one of 18 families, or designated non-core (without family), based on previously designated family of gene homologues.

\section{Bit-score plots}

Bit-scores were calculated using Geneious Pro 6.1.4 (Biomatters Ltd., Auckland, New Zealand) to compare individual CyCMV Mauritius or RhCMV 180.92 ORFs to homologous ORFs of CyCMV Ottawa (accession: JN227533), RhCMV 180.92 (accession: DQ120516), and RhCMV 68-1 (accession: AY186194) found using BLAST-P (NCBI) using a BLOSUM62 matrix, gap opening cost of 11, gap extension cost of one and a maximum $\mathrm{E}$ value of $10^{-1}$. Calculated scores for individual ORF were plotted as seen previously [18] as a scatterplot comparing the bit-score for CyCMV MauritiusCyCMV Ottawa, CyCMV Mauritius -RhCMV 180.92 and CyCMV Mauritius - RhCMV 68-1 to each other.

\section{Gross genome comparison}

CyCMV Mauritius has been deposited in the GenBank database (accession: KP796148). Other Genomes and annotations were obtained from GenBank using available strains as follows: CyCMV Ottawa (accession: JN227533), RhCMV 180.92 (accession: DQ120516), and RhCMV 68-1 (accession: AY186194). Comparisons were also made with human CMV strains [HCMV Towne (accession: AY315197), HCMV AD169 (accession: X17403) and HCMV HAN1 (accession: JX512199)], chimpanzee CMV Heberling (accession: AF480884), Baboon cytomegalovirus OCOM4-37 (accession: AC090446), Cercopithecine herpesvirus 5 (GMCMV) Colburn (accession: FJ483969), Cercopithecine herpesvirus 5 (GMCMV) strain 2715 (accession: FJ483968), Aotine herpesvirus 1 strain S34E (accession: FJ483970), and Saimiriine herpesvirus 3 SqSHV (accession: FJ483967). Genome identity between CMV strains was determined using global alignment with free end gaps set at a cost matrix of $65 \%$ similarity $(5.0 /-4.0)$, a gap open penalty of 12 and a gap extension penalty of three with automatic sequence direction determination on Geneious 6.1.4 (Biomatters Ltd., Auckland, New Zealand). Whole genome alignments were generated using progressive MAUVE multiple genome alignment algorithm [21] available in Geneious 6.1.4 (Biomatters Ltd., Auckland, New Zealand).

\section{Phylogenetic analysis}

Phylogenetic analysis was carried out with Geneious Pro 6.1.4 (Biomatters Ltd., Auckland, New Zealand). Alignments were created using MAFFT version 7.017 [47] with a scoring matrix of 200PAM/k = 2 a Gap open penalty of 1.53 and offset value of 0.123 . An appropriate phylogenetic model was selected for each tree using JModel test 2.1.7 [48]. Most trees were generated with the MrBayes plugin for Geneious Pro 6.1.4 [49] after 1100000 iterations and a burn-in of 110 000. The individual ORFs or whole genome sequences of CyCMV Mauritius, CyCMV Ottawa (accession: JN227533), RhCMV 68-1 (accession: AY186194), and RhCMV 180.92 (accession: DQ120516), were compared with the outgroup HCMV AD169 (accession: FJ527563) (Fig. 2), Homo Sapiens PTGS2 (accession: BAA05698) (Fig. 6a), Homo Sapiens CXCL5 (accession: CR457428) (Fig. 6b), or Homo Sapiens TNFRSF14X1 (trimmed CDS from accession: XM_011542383) (Fig. 6c). The 12 CMV full genome tree (Fig. 7) was generated using MrBayes 3.2.4 run in MPI on the SciNet supercomputer at the University of Toronto [50] using a generalized time reversible (GTR) tree with proportion invariant sites and gamma distribution. Genomes utilized were selected from fully sequenced CMV genomes at the time of publication due to similar organization. CMVs included were free from large rearrangements or inverted regions with respect to CyCMV Mauritius as determined from alignments using progressive MAUVE multiple genome alignment algorithm [21] in Geneious 6.1.4 (Biomatters Ltd., Auckland, New Zealand). RCMV Maastricht (Rat) (accession: AF232689) was used as the out-group.

\section{Availability of data and materials}

The genome sequence for CyCMV Mauritius has been deposited in the GenBank database (accession no. KP796148). 


\section{Additional files}

Additional file 1: Figure S1. Map of ORFs in CyCMV Mauritius genome CyCMV Mauritius encodes 290 putative ORFs that are annotated by gene name and colour coded based on gene families. Of the CyCMV Mauritius ORFs, 268 (92\%) share homologues with CyCMV Ottawa, 239 share homologues with RhCMV 68-1 (82\%), and 158 (54\%) share homologs with HCMV strains. CyCMV Mauritius like RhCMV but unlike CyCMV Ottawa or HCMV contains ORFs with homology to COX-2. CyCMV ORFs with an HCMV homologue are annotated by "Cy" followed by the HCMV name. Arrowheads indicate the directions of the ORFs. Core genes are herpes virus core genes. (PDF $4584 \mathrm{~kb}$ )

Additional file 2: Table S1. ORFs of CyCMV Mauritius. Footnotes: 1 Functions annotated based on studies of HCMV [52] unless otherwise indicated. 2 Nearest homologous HCMV gene based on Bit score using BLASTP search. Strain of HCMV gene indicated with footnote. 3 Based on function of RhCMV68-1 homologue [19]. 4 Based on function of CyCMV Ottawa homologue [18]. 5 Based on studies of HCMV Towne-BAC [54]. 6 Inferred through function of homologous proteins [52]. 8 HCMV strain AD169. 9 HCMV strain AF1. 10 HCMV strain ASM72. 11 HCMV strain C154. 12 HCMV strain C425, 13 HCMV strain CINCY + Towne. 14 HCMV strain Coz. 15 HCMV strain David. 16 HCMV strain FRCMV-14L. 17 HCMV strain GSV6. 18 HCMV strain GSV9. 19 HCMV strain HAN1. 20 HCMV strain HAN13. 21 HCMV strain HAN16. 22 HCMV strain HAN20. 23 HCMV strain HAN3. 24 HCMV strain HAN38. 25 HCMV strain HKS40. 26 HCMV strain I10. 27 HCMV strain IS17. 28 HCMV strain JHC. 29 HCMV strain JP. 30 HCMV strain L2. 31 HCMV strain Merlin. 32 HCMV strain RK (Human Herpesvirus 7). 33 HCMV strain TB40/E. 34 HCMV strain Toledo. 35 HCMV strain Towne. 36 HCMV strain TR. 37 HCMV strain U11. 38 HCMV strain U8. 39 HCMV strain VR1814. 40 HCMV strain 35. 41 HCMV strain 66. 42 HCMV strain 452. 43 HCMV strain 553. 44 HCMV strain 3157. 45 HCMV strain 3301. 46 HCMV strain 5234. 47 HCMV strain 401058. 48 HCMV strain 26M. 49 HCMV strain 51C. (PDF 158 kb)

Additional file 3: Figure S2. Break down of two-dimensional bit-score plots between CMV Mauritius-RhCMV 68.1 versus CyCMV MauritiusRhCMV 180.92 by ORF function. Graphs represent comparisons of ORF homologue bit-scores across three strains simultaneously. ORFs are coloured according to known function. Individual ORF may have several functions and are present in multiple plots. Immune ORFs are involved in host immune regulation or evasion, entry ORFs are known cell entry factors, temperance ORFs are involved in temperance of viral growth, membrane ORFs are presented in cell membrane on infected cells or in virions, DNA/nuclear are known to interact with DNA or shuttle to the cell nucleus, capsid ORFs form the virus capsid, tegument proteins are found in the viral tegument, the function of ORFs with other known function vary, and the function is unknown at the time of writing for ORFs plotted as unknown. ORFs are annotated according to CyCMV Mauritius names except for ORFs of unknown function or membrane ORF where some ORF are left unlabelled. (PDF $49 \mathrm{~kb}$ )

Additional file 4: Figure S3. Break down of two-dimensional bit-score plots between CyCMV Mauritius-CyCMV Ottawa versus RhCMV 68-1-RhCMV 180.92 by ORF function. Graphs represent comparisons of ORF homologue bit-scores across three strains simultaneously. ORFs are coloured according to known function. Individual ORF may have several functions and are present in multiple plots. Immune ORFs are involved in host immune regulation or evasion, entry ORFs are known cell entry factors, temperance ORFs are involved in temperance of viral growth, membrane ORFs are presented in cell membrane on infected cells or in virions, DNA/nuclear are known to interact with DNA or shuttle to the cell nucleus, capsid ORFs form the virus capsid, tegument proteins are found in the viral tegument, the function of ORFs with other known function vary, and the function is unknown at the time of writing for ORFs plotted as unknown. ORFs are annotated according to CyCMV Mauritius names except for ORFs of unknown function or membrane ORF where some ORF are left unlabelled. (PDF 52 kb)

Additional file 5: Figure S4. Break down of two-dimensional bit-score plots between CyCMV Mauritius-CyCMV Ottawa versus CyCMV MauritiusRhCMV 180.92 by ORF function. Graphs represent comparisons of ORF homologue bit-scores across three strains simultaneously. ORFs are coloured according to known function. Individual ORF may have several functions and are present in multiple plots. Immune ORFs are involved in host immune regulation or evasion, entry ORFs are known cell entry factors, temperance ORFs are involved in temperance of viral growth, membrane ORFs are presented in cell membrane on infected cells or in virions, DNA/nuclear are known to interact with DNA or shuttle to the cell nucleus, capsid ORFs form the virus capsid, tegument proteins are found in the viral tegument, the function of ORFs with other known function vary, and the function is unknown at the time of writing for ORFs plotted as unknown. ORFs are annotated according to CyCMV Mauritius names except for ORFs of unknown function or membrane ORF where some ORF are left unlabelled. (PDF $50 \mathrm{~kb}$ )

Additional file 6: Figure S5. Break down of two-dimensional bit-score plots between CyCMV Mauritius-CyCMV Ottawa versus CyCMV MauritiusRhCMV 68-1 by ORF function. Graphs represent comparisons of ORF homologue bit-scores across three strains simultaneously. ORFs are coloured according to known function. Individual ORF may have several functions and are present in multiple plots. Immune ORFs are involved in host immune regulation or evasion, entry ORFs are known cell entry factors, temperance ORFs are involved in temperance of viral growth, membrane ORFs are presented in cell membrane on infected cells or in virions, DNA/nuclear are known to interact with DNA or shuttle to the cell nucleus, capsid ORFs form the virus capsid, tegument proteins are found in the viral tegument, the function of ORFs with other known function vary, and the function is unknown at the time of writing for ORFs plotted as unknown. ORFs are annotated according to CyCMV Mauritius names except for ORFs of unknown function or membrane ORF where some ORF are left unlabelled. (PDF $50 \mathrm{~kb}$ )

Additional file 7: Figure S6. Plot of bit-scores of ORFs across the CyCMV Mauritius genome. ORFs of CyCMV Mauritius are ordered as seen in CyCMV Mauritius genome. Bit-score is plotted for each ORF between CyCMV Mauritius and CyCMV Ottawa, RhCMV 68-1, and RhCMV 180.92. Absence of the indicated gene from one or more compared genomes results in a bit-score of zero. Values are indicated as the means \pm standard deviation for each gene. (PDF 147 kb)

Additional file 8: Figure S7. Break down of two-dimensional bit-score plots between CMV Mauritius-CyCMV Ottawa versus RhCMV 68-1-RhCMV 180.92 by ORF family. Graphs represent comparisons of ORF homologue bit-scores across three strains simultaneously. ORFs are coloured according to ORF family. CyCMV Mauritius names for ORF labels. (PDF 57 kb)

Additional file 9: Figure S8. Break down of two-dimensional bit-score plots between CyCMV Mauritius-RhCMV 68-1 versus CyCMV MauritiusRhCMV 180.92 by ORF family. Graphs represent comparisons of ORF homologue bit-scores across three strains simultaneously. ORFs are coloured according to ORF family. CyCMV Mauritius names for ORF labels. (PDF $56 \mathrm{~kb}$ )

Additional file 10: Figure S9. Break down of two-dimensional bit-score plots between CMV Mauritius-CyCMV Ottawa versus CyCMV MauritiusRhCMV 180.92 by ORF family. Graphs represent comparisons of ORF homologue bit-scores across three strains simultaneously. ORFs are coloured according to ORF family. CyCMV Mauritius names used for ORF labels. (PDF $57 \mathrm{~kb}$ )

Additional file 11: Figure S10. Break down of two-dimensional bit-score plots between CMV Mauritius-RhCMV 68-1 versus CyCMV Mauritius-CyCMV Ottawa by ORF family. Graphs represent comparisons of ORF homologue bit-scores across three strains simultaneously. ORFs are coloured according to ORF family. CyCMV Mauritius names used for ORF labels. (PDF 57 kb)

\section{Competing interests}

The authors declare that they have no competing interest.

\section{Author's contributions}

JNHR, AKM, DOW, and KSM designed research; JNHR, AKM, APNA, MD, JKC, RP, $J F$, and PS performed research: JNHR, JMA, BJE, and KSM analyzed data; MB, and PS contributed reagents/materials/analytical tools; JNHR, JMA, BJE, and KSM wrote the paper. All authors read and approved the final manuscript. 


\section{Acknowledgments}

This work was supported by the Canadian Institutes for Health Research (ClHR, grant number 107743). A CIHR Doctoral research award to A.K.M, an OHTN Junior Investigator Development Award to D.O.W and an OHTN Career Scientist Award to K.S.M. and postdoctoral fellowship to A.P.A are acknowledged. Computations were performed on the general purpose cluster (GPC) supercomputer at the SciNet HPC Consortium. SciNet is funded by: the Canada Foundation for Innovation under the auspices of Compute Canada; the Government of Ontario; Ontario Research Fund - Research Excellence; and the University of Toronto. The authors thank the veterinary and technical staff at the NHP colony of Health Canada for their help. The authors wish to thank the late Dr Allan J Baker of the Royal Ontario Museum for his thoughts on refining macaque evolution with co-evolved viruses and Catia Perciani for critical reading of the manuscript.

\section{Author details}

'Department of Immunology, University of Toronto, Toronto M5S 1A8ON, Canada. ${ }^{2}$ Department of Medicine, University of Toronto, Toronto M5S 1 A8ON, Canada. ${ }^{3}$ Department of Microbiology, Mount Sinai Hospital, Toronto M5G 1X5ON, Canada. ${ }^{4}$ Department of Computer Science, University of Toronto, Toronto M5S 1A8ON, Canada. ${ }^{5}$ National HIV \& Retrovirology Laboratories, Public Health Agency of Canada, Ottawa K1A OK9ON, Canada. ${ }^{6}$ Scientific Services Division, Health Products \& Food Branch, Health Canada, Ottawa K1A OL2ON, Canada. ${ }^{7}$ Biology Department, McMaster University, Life Sciences Building, 1280 Main Street West, Hamilton L8S 4K1ON, Canada. ${ }^{8}$ Section of Infectious Diseases, Department of Internal Medicine, University of Manitoba, 745 Bannatyne Ave, Winnipeg R3E 0J9MB, Canada. ${ }^{9}$ Present Address: Canadian Science Centre for Human and Animal Health, National Centre for Foreign Animal Disease, 1015 Arlington Street, Winnipeg R3E 3M4MB, Canada. ${ }^{10}$ Present Address: University of Manitoba, Basic Medical Sciences Building, Room 501, 745 Bannatyne Ave., Winnipeg R3E 0J9MB, Canada.

\section{Received: 9 October 2015 Accepted: 14 March 2016}

\section{Published online: 05 April 2016}

\section{References}

1. [No Authors Listed]. Demands for rhesus monkeys in biomedical research: a workshop report. ILAR J. 2003:44(3):222-35.

2. Antony JM, MacDonald KS. A critical analysis of the cynomolgus macaque, Macaca fascicularis, as a model to test HIV-1/SIV vaccine efficacy. Vaccine. 2015; 33(27):3073-83

3. Kanthaswamy S, Ng J, Trask JS, George DA, Kou AJ, Hoffman LN, Doherty TB, Houghton P, Smith DG. The genetic composition of populations of cynomolgus macaques (Macaca fascicularis) used in biomedical research. J Med Primatol. 2013:42(3):120-31.

4. Delson E. Fossil Macaques, Phyletic Relationships and a Scenario of Deployment. In: Lindburg DG, editor. The Macaques: Studies in ecology, behaviour, and evolution. New York: Van Nostrand-Reinhold; 1980. p. 10-30.

5. Trask JAS, Garnica WT, Smith DG, Houghton P, Lerche N, Kanthaswamy S. Single-Nucleotide Polymorphisms Reveal Patterns of Allele Sharing Across the Species Boundary Between Rhesus (Macaca mulatta) and Cynomolgus (M. fascicularis) Macaques. Am J Primatol. 2013;75(2):135-44.

6. Creager HM, Becker EA, Sandman KK, Karl JA, Lank SM, Bimber BN, Wiseman RW, Hughes AL, O'Connor SL, O'Connor DH. Characterization of full-length MHC class II sequences in Indonesian and Vietnamese cynomolgus macaques. Immunogenetics. 2011;63(9):611-8.

7. Davison A, Holto M, DOlan A, Dargan D, Gatherer D, Haywayrd G. Comparatve Genomics of Primate Cytomegaloviruses. In: Reddehase MJ, editor. Cytomegaloviruses: From Molecular Pathogenesis to Intervention. Norfolk: Caister Academic Publishing; 2013. p. 1-22. vol. 1.

8. Leendertz FH, Deckers M, Schempp W, Lankester F, Boesch C, Mugisha L, Dolan A, Gatherer D, McGeoch DJ, Ehlers B. Novel cytomegaloviruses in free-ranging and captive great apes: phylogenetic evidence for bidirectional horizontal transmission. J Gen Virol. 2009;90(Pt 10):2386-94.

9. Alcendor DJ, Zong J, Dolan A, Gatherer D, Davison AJ, Hayward GS. Patterns of divergence in the $V C X C L$ and $V G P C R$ gene clusters in primate cytomegalovirus genomes. Virology. 2009:395(1):21-32.

10. Oxford KL, Eberhardt MK, Yang KW, Strelow L, Kelly S, Zhou SS, Barry PA. Protein coding content of the UL)b' region of wild-type rhesus cytomegalovirus. Virology. 2008;373(1):181-8.
11. Davison AJ. Comparative analysis of the genomes. In: Arvin A, Mocarski CFG, editors. Human Herpesviruses: Biology, Therapy, and Immunoprophylaxis. Cambridge: Cambridge University Press; 2007.

12. Grose C. Pangaea and the Out-of-Africa Model of Varicella-Zoster Virus Evolution and Phylogeography. J Virol. 2012;86(18):9558-65.

13. Kolb AW, Ané C, Brandt CR. Using HSV-1 Genome Phylogenetics to Track Past Human Migrations. PLoS One. 2013;8(10), e76267.

14. Smith AL, Black DH, Eberle R. Molecular evidence for distinct genotypes of monkey $B$ virus (herpesvirus simiae) which are related to the macaque host species. J Virol. 1998;72(11):9224-32.

15. Chou S. Reactivation and Recombination of Multiple Cytomegalovirus Strains from Individual Organ Donors. J Infect Dis. 1989;160(1):11-5.

16. Lees DN, Baskerville A, Cropper LM, Brown DW. Herpesvirus simiae (B virus) antibody response and virus shedding in experimental primary infection of cynomolgus monkeys. Lab Anim Sci. 1991;41(4):360-4.

17. Marsh AK, Ambagala AP, Perciani CT, Russell JN, Chan JK, Janes M, Antony $J M$, Pilon R, Sandstrom P, Willer DO et al. Examining the species-specificity of rhesus macaque cytomegalovirus (RhCMV) in cynomolgus macaques. PLoS One. 2015;10(3), e0121339.

18. Marsh AK, Willer DO, Ambagala AP, Dzamba M, Chan JK, Pilon R, Fournier J, Sandstrom P, Brudno M, MacDonald KS. Genomic sequencing and characterization of cynomolgus macaque cytomegalovirus. J Virol. 2011; 85(24):12995-3009.

19. Hansen SG, Strelow LI, Franchi DC, Anders DG, Wong SW. Complete sequence and genomic analysis of rhesus cytomegalovirus. J Virol. 2003; 77(12):6620-36.

20. Rivailler P, Kaur A, Johnson RP, Wang F. Genomic sequence of rhesus cytomegalovirus 180.92: insights into the coding potential of rhesus cytomegalovirus. J Virol. 2006;80(8):4179-82.

21. Darling AC, Mau B, Blattner FR, Perna NT. Mauve: multiple alignment of conserved genomic sequence with rearrangements. Genome Res. 2004; 14(7):1394-403.

22. Lilja AE, Chang WL, Barry PA, Becerra SP, Shenk TE. Functional genetic analysis of rhesus cytomegalovirus: Rh01 is an epithelial cell tropism factor. J Virol. 2008:82(5):2170-81.

23. Wong WSW, Nielsen R. Detecting selection in noncoding regions of nucleotide sequences. Genetics. 2004;167(2):949-58.

24. Ohta T. The Nearly Neutral Theory of Molecular Evolution. Annu Rev Ecol Syst. 1992;23:263-86.

25. Mcvoy MA, Adler SP. Human Cytomegalovirus DNA Replicates after Early Circularization by Concatemer Formation, and Inversion Occurs within the Concatemer. J Virol. 1994;68(2):1040-51.

26. Assaf BT, Mansfield KG, Strelow L, Westmoreland SV, Barry PA, Kaur A. Limited dissemination and shedding of the UL128 complex-intact, UL/b'defective rhesus cytomegalovirus strain 180.92. J Virol. 2014;88(16):9310-20.

27. Ryckman BJ, Rainish BL, Chase MC, Borton JA, Nelson JA, Jarvis MA, Johnson DC. Characterization of the human cytomegalovirus $\mathrm{gH} / \mathrm{gL} / \mathrm{LL}$ 128-131 complex that mediates entry into epithelial and endothelial cells. J Virol. 2008:82(1):60-70.

28. Nicholas J. Evolutionary aspects of oncogenic herpesviruses. Molecular pathology : MP. 2000;53(5):222-37.

29. Wiertz EJ, Jones TR, Sun L, Bogyo M, Geuze HJ, Ploegh HL. The human cytomegalovirus US11 gene product dislocates MHC class I heavy chains from the endoplasmic reticulum to the cytosol. Cell. 1996;84(5):769-79.

30. Rue CA, Jarvis MA, Knoche AJ, Meyers HL, DeFilippis VR, Hansen SG, Wagner M, Fruh K, Anders DG, Wong SW et al. A cyclooxygenase-2 homologue encoded by rhesus cytomegalovirus is a determinant for endothelial cell tropism. J Virol. 2004;78(22):12529-36.

31. Golden MP, Hammer SM, Wanke CA, Albrecht MA. Cytomegalovirus vasculitis. Case reports and review of the literature. Medicine (Baltimore). 1994;73(5):246-55

32. Arslan F, Batirel A, Mert A, Ozer S. Cytomegalovirus (CMV)-related cutaneous necrotizing vasculitis: case report and literature review. Braz I Infect Dis. 2012;16(5):482-5.

33. Cohen P, Guillevin L. Vascularites associées aux infections virales. Presse Med. 2004;33:1371-84.

34. Yi HA, Kim MS, Jang SY, Lee YM, Ahn JH, Lee CH. Cellular signals involved in cyclooxygenase-2 expression induced by human cytomegalovirus. Virus Res. 2009;146(1-2):89-96

35. Penfold MET, Schmidt TL, Dairaghi DJ, Barry PA, Schall TJ. Characterization of the Rhesus Cytomegalovirus US28 Locus. J Virol. 2003;77(19):10404-13. 
36. Neubauer SR. Characterization of the genome of baboon cytomegalovirus strain (OCOM4-37) isolated from the olive baboon, Papio cynocephalus anubis. Ph.D Thesis. 2011. Oklahoma State University, Stillwater, OK.

37. Rogers J, Gibbs RA. Comparative primate genomics: emerging patterns of genome content and dynamics. Nat Rev Genet. 2014;15(5):347-59.

38. Hansen SG, Sacha JB, Hughes CM, Ford JC, Burwitz BJ, Scholz I, Gilbride RM, Lewis MS, Gilliam AN, Ventura AB et al. Cytomegalovirus vectors violate CD8 + T cell epitope recognition paradigms. Science. 2013;340(6135):1237874.

39. Gerna G, Percivalle E, Sarasini A, Baldanti F, Revello MG. The attenuated Towne strain of human cytomegalovirus may revert to both endothelial cell tropism and leuko- (neutrophil- and monocyte-) tropism in vitro. J Gen Virol. 2002;83(Pt 8):1993-2000.

40. Schleiss MR. Developing a Vaccine against Congenital Cytomegalovirus (CMV) Infection: What Have We Learned from Animal Models? Where Should We Go Next? Futur Virol. 2013:8(12):1161-82.

41. Tosi AJ, Coke CS. Comparative phylogenetics offer new insight into the biogeographic history of Macaca fascicuaris and the origin of the Mauritian macaque. Mol Phylogenet Evol. 2007;42:498-504.

42. Tosi AJ, Morales JC, Melnick DJ. Comparison of $Y$ chromosome and mtDNA phylogenies leads to unique inferences of macaque evolutionary history. Mol Phylogenet Evol. 2000;17(2):133-44.

43. Numazaki $\mathrm{K}$, Ikehata $\mathrm{M}$, Chiba S. Subtyping of cytomegalovirus strains obtained from immunocompetent children. In vivo. 2000;14(6):745-6.

44. Jacobs JP, Jones CM, Baille JP. Characteristics of a human diploid cell designated MRC-5. Nature. 1970;227(5254):168-70.

45. Zerbino DR, Birney E. Velvet: algorithms for de novo short read assembly using de Bruijn graphs. Genome Res. 2008;18(5):821-9.

46. Borst EM, Messerle M. Analysis of human cytomegalovirus orilyt sequence requirements in the context of the viral genome. J Virol. 2005;79(6):3615-26.

47. Katoh K, Misawa K, Kuma K, Miyata T. MAFFT: a novel method for rapid multiple sequence alignment based on fast Fourier transform. Nucleic Acids Res. 2002;30(14):3059-66.

48. Darriba D, Taboada GL, Doallo R, Posada D. jModelTest 2: more models, new heuristics and parallel computing. Nat Methods. 2012;9(8):772.

49. Huelsenbeck JP, Ronquist F. MRBAYES: Bayesian inference of phylogenetic trees. Bioinformatics. 2001;17(8):754-5.

50. Gruner CLD, Groer L, Peltier R, Bunn N, Craig M, Henriques T, Dempsey J, Yu C-H, Chen J, Dursi LJ, Chong J, Northrup S, Pinto J, Knecht N, Van Zon R. SciNet: Lessons Learned from Building a Power-efficient Top-20 System and Data Centre. J Phys Conf Ser. 2010;256(1):012026.

51. Dunn W, Chou C, Li H, Hai R, Patterson D, Stolc V, Zhu H, Liu F. Functional profiling of a human cytomegalovirus genome. Proc Natl Acad Sci U S A. 2003;100(24):14223-8.

52. Mocarski ES, Shenk T, Pass R. Cytomegaloviruses. In: Knipe DM, Howley P, editors. Feilds Virology. Philadelphia: Lippincott, Williams \& Wilkins; 2007. p. 2702-57. vol. 2, 5 edn

53. Arnolds KL, Lares AP, Spencer JV. The US27 gene product of human cytomegalovirus enhances signaling of host chemokine receptor CXCR4. Virology. 2013;439(2):122-31.

54. Omoto S, Mocarski ES. Cytomegalovirus UL91 is essential for transcription of viral true late (gamma2) genes. J Virol. 2013;87(15):8651-64.

\section{Submit your next manuscript to BioMed Central and we will help you at every step:}

- We accept pre-submission inquiries

- Our selector tool helps you to find the most relevant journal

- We provide round the clock customer support

- Convenient online submission

- Thorough peer review

- Inclusion in PubMed and all major indexing services

- Maximum visibility for your research

Submit your manuscript at www.biomedcentral.com/submit
Biomed Central 